\title{
Estresse e apoio social em cuidadores de crianças com paralisia cerebral ${ }^{1}$
}

\author{
Alyne Kalyane Câmara de Oliveira ${ }^{a}$, Thelma Simóes Matsukura ${ }^{b}$

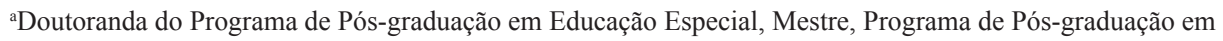 \\ Terapia Ocupacional, Universidade Federal de São Carlos - UFSCar, São Carlos, SP, Brasil \\ bProfessora Associada do Departamento de Terapia Ocupacional e dos Programas de Pós-graduação em Terapia \\ Ocupacional e Educação Especial, Universidade Federal de São Carlos - UFSCar, São Carlos, SP, Brasil
}

\begin{abstract}
Resumo: O estudo objetivou descrever níveis de estresse e apoio social percebido de cuidadores de crianças com paralisia cerebral ( $\mathrm{PC}$ ), assim como investigar relações entre estresse e apoio social e variáveis relativas aos cuidadores, ao ambiente e às crianças, a saber: número de filhos, escolaridade do cuidador, renda familiar, temperamento e nível motor da criança. Participaram 50 crianças com PC com idade entre 3 e 7,5 anos, os respectivos 50 cuidadores e 25 profissionais da reabilitação, que tratam as crianças em instituições de saúde do interior de São Paulo. Os instrumentos de medida utilizados foram o Sistema de Classificação da Função Motora Grossa para Paralisia Cerebral, o Inventário de Sintomas de Stress para Adultos de Lipp, o Questionário de Suporte Social e uma ficha de identificação dos participantes. Os dados foram analisados por meio de estatística descritiva e inferencial, com os testes Qui-quadrado, Exato de Fisher, Mann-Whitney, Kruskal-Wallis e Odds-Ratio. Os resultados apontaram estresse entre os cuidadores participantes (66\%), com predominância da fase de resistência $(93,9 \%)$ e sintomas psicológicos $(69,7 \%)$, baixo apoio social percebido pelos cuidadores, concomitante a uma adequada satisfação com $\mathrm{o}$ apoio recebido, assim como relações significativas entre $\mathrm{o}$ apoio social versus estresse $(p=0,017)$ e escolaridade versus apoio social $(\mathrm{p}=0,037)$. Os dados permitem a análise das relações entre as variáveis investigadas e acerca do impacto de se ter uma criança com PC na família sobre o bem-estar físico, emocional e psicológico dos cuidadores, além de oferecer subsídios para a elaboração de estratégias em diferentes níveis de atenção às famílias de crianças com deficiência.
\end{abstract}

Palavras-chave: Cuidadores, Apoio Social, Estresse Psicológico, Paralisia Cerebral, Terapia Ocupacional.

\section{Stress and social support in caregivers of children with cerebral palsy}

\begin{abstract}
In this study, we aimed to describe the levels of stress and perceived social support for caregivers of children with cerebral palsy (CP), as well as investigate the relationship between stress, social support, and variables related to caregivers, the environment and children, namely: the number of children, education level of caregivers, family income, behavior, and the child's motor level. This study comprised 50 children with CP between 3 and 7.5 years old, their 50 caregivers, and 25 rehabilitation professionals who care for children in health institutions from the countryside of São Paulo state, Brazil. The following measuring instruments were used: the Gross Motor Function Classification System for Cerebral Palsy, the Lipp's Inventory of Stress Symptoms in Adults, the Social Support Questionnaire, and a form identifying the participants. Data were analyzed using descriptive and inferential statistics by the following tests: Chi-square, Fisher exact, Mann-Whitney, Kruskal-Wallis, and Odds Ratio. The results showed stress among the participating caregivers (66\%), with predominance of the resistance phase (93.9\%) and psychological symptoms (69.7\%), low perceived social support for caregivers, concomitant with an adequate satisfaction with the support received, as well as significant relationships of stress versus social support $(\mathrm{p}=0.017)$ and education level versus social support $(\mathrm{p}=0.037)$. The data allow analysis of the relationship between the variables
\end{abstract}

Autor para correspondência: Alyne Kalyane Câmara de Oliveira, Laboratório de Saúde Mental, Departamento de Terapia Ocupacional, Universidade Federal de São Carlos, Rod. Washington Luís, km. 235, CEP: 13565-905, São Carlos, SP, Brasil, e-mail: alynekoliveira@ hotmail.com Recebido em 2/5/2012; $1^{\text {a }}$ Revisão em 17/9/2012; 2 ${ }^{a}$ Revisão em 2/10/2012; Aceito em: 4/11/2012. 
investigated and about the impact of having a child with CP in the family regarding the physical, emotional and psychological well-being of caregivers, besides providing subsidies to think of strategies at different levels of care for families of children with disabilities.

Keywords: Caregivers, Social Support, Stress, Cerebral Palsy, Occupational Therapy.

\section{Introdução}

A partir de consideraçóes acerca da importância dos diversos contextos que envolvem o desenvolvimento infantil e o resultado de intervençóes junto a crianças com deficiências, tornou-se mais frequente a busca pela compreensão do papel dos cuidadores, do impacto da doença e dos cuidados dispensados às crianças, assim como sobre os processos de adaptação familiar e diferentes variáveis que podem mediar o enfrentamento das demandas do dia a dia (MATSUKURA et al., 2007).

Pesquisas têm abordado as reaçóes dos familiares em relação ao reconhecimento do diagnóstico, como a apresentada por Ferrari e Morete (2004) ao observarem que o impacto da notícia da condição da criança mobiliza várias reaçóes emocionais no familiar, que deverá se reorganizar psiquicamente para o processo de cuidar do filho com comprometimento. Ainda em termos de reaçóes e sentimentos envolvidos, a família pode passar por estágios, a partir do momento em que recebe o diagnóstico da criança, como as etapas de choque, negação, luto, tristeza e ajustamento (DANTAS et al., 2010).

Em relação às dúvidas sobre os cuidados com a criança e expectativas sobre o futuro dela, estudos têm indicado que existem demandas comuns às famílias, independentemente do tipo de deficiência da criança, como a necessidade de orientaçôes, informações e serviços, e outras que parecem ser específicas e relacionadas às dificuldades das crianças e à etapa do desenvolvimento infantil em que se encontram (HIRATUKA, 2009; MATSUKURA; SIME, 2008; HEIMAN, 2002).

Nessa direção, a literatura também tem destacado o impacto da deficiência infantil como sobrecarga sobre o processo de cuidar dessa criança (CAMARGOS et al., 2009), as mudanças nos papéis ocupacionais dos familiares (CROWE; VANLEIT; BERGHMANS, 2000) sobre o cotidiano do cuidador (STENER-VICTORIN et al., 2010; VIEIRA et al., 2008) e, sobretudo, a questão da qualidade de vida e aspectos da saúde física/mental desses cuidadores (BYRNE et al., 2010; DAVIS et al., 2009).

No caso dos cuidadores de crianças com diagnóstico de paralisia cerebral (PC), que necessitam participar mais ativamente do cuidado dessas crianças com condiçôes crônicas, vários aspectos da vida dos cuidadores podem ser impactados (LIN; ZEBRACK, 2004).

A PC compreende um grupo de desordens do movimento e da postura, causando limitaçóes funcionais atribuídas a distúrbios não progressivos que ocorrem durante o desenvolvimento do cérebro fetal ou infantil (ROSEMBAUM et al., 2007). Embora a lesão primária no cérebro não seja progressiva, a condição é permanente e muitas das consequências clínicas secundárias são adquiridas e são progressivas ao longo do tempo (KOKKONEN et al., 2001).

Dos danos resultantes de lesões cerebrais, as crianças com PC podem apresentar comprometimentos diversos, a depender do alcance da lesão no Sistema Nervoso Central, geralmente associados à gravidade da sequela e à idade da criança acometida (MANCINI et al., 2004). O distúrbio motor é a base do quadro clínico, mas a condição pode se associar com diferentes combinaçóes: com transtornos de linguagem, auditivos, visuais, cognitivos e comportamentais, com dificuldade de alimentação e de percepção, com déficits sensoriais, problemas odontológicos, crises epilépticas e deficiência intelectual, entre outros (ROSEMBAUM et al., 2007).

Relacionando todas essas manifestações clínicas (desordens motoras e distúrbios associados) da PC ao impacto do processo de cuidar de crianças com deficiência, o estudo qualitativo de Davis et al. (2009), realizado através de entrevistas semiestruturadas sobre a qualidade de vida de 24 mães e 13 pais de crianças e adolescentes com PC, na faixa etária entre 3 e 7 , entre 8 e 12 e entre 13 e 18 anos, com diferentes níveis de comprometimento, destaca a influência que cuidar de uma criança com deficiência tem no bem-estar físico e social, na liberdade, na independência e na estabilidade financeira dos cuidadores, além do impacto no bem-estar da unidade familiar.

O estresse dos cuidadores, como uma reação do organismo com componentes psicológicos, físicos, mentais e hormonais, pode ser considerado um dos aspectos presentes na vida dos familiares de crianças com deficiência. Diz-se que há estresse na presença de diferença acentuada entre as demandas externas e a 
avaliaçáo da pessoa sobre sua capacidade de responder a elas. É um processo no qual o indivíduo percebe e responde a eventos julgados danosos, desafiadores ou ameaçadores, podendo originar alteraçóes fisiológicas, psicológicas, cognitivas, comportamentais e emocionais (MATSUKURA et al., 2007; LIPP, 2000).

Lipp (2003) esclarece que o estresse surge em um processo de etapas, nas quais a sintomatologia e a forma de manejo são diferenciadas conforme a fase do estresse observada. De acordo com o modelo proposto pela autora, são quatro as fases do estresse (alarme, resistência, quase exaustão e exaustáo), sendo que as distinçóes entre elas se fazem pela duração da ação do agente estressor, assim como pelo surgimento de sintomas físicos e/ou psicológicos (LIPP, 2000).

Lin e Zebrack (2004) apontam que o processo de cuidar de uma criança com deficiência juntamente com o aumento das responsabilidades que a função requer podem levar ao cansaço, isolamento, sobrecarga e consequente estresse dos cuidadores. E tratando-se de pais de crianças com PC, estudos indicam que por vezes eles apresentam elevados níveis de estresse quando comparados a pais de crianças sem comprometimentos (GLENN et al., 2008; RAINA et al., 2005; MANUEL et al., 2003).

Apesar de, enquanto grupo, esses familiares estarem sujeitos a aspectos como maior sobrecarga, ansiedade e níveis de estresse, como apresentado pela literatura, cada familiar e/ou cuidador lida de forma diferente na maneira como percebem os desafios de ter uma criança com deficiência na família (CALDERON; GREENBERG, 1999; CANNING; HARRIS; KELLEHER, 1996), ideia essa reforçada por Raina et al. (2005) ao localizarem variação considerável na forma como uma grande amostra de cuidadores de crianças com PC (468 cuidadores que vivem no Canadá) se adaptam aos fatores estressantes e demandas geradas pelo processo de ter uma criança com deficiência. Nunes (2007), ao buscar identificar o perfil dos cuidadores de crianças com PC de 4 a 12 anos de idade e os agentes estressores presentes na realidade dessas famílias, quando comparadas a um grupo controle de cuidadores de crianças típicas, encontrou uma tendência de esses familiares apresentarem mais problemas na saúde física, emocional e prejuízo na vida social; não obstante, a autora aponta a necessidade de identificar a variabilidade das respostas frente aos estressores.

Como destacado, os estressores têm apenas a capacidade de desencadear a reação ao estresse, que por sua vez será mediado pela forma como cada pessoa suscita condiçóes para o manejo da situação adversa (ARAGÃO et al., 2009). O modo como a pessoa irá lidar com a situação estressora dependerá da forma de seu enfrentamento ou recursos de coping, que envolvem a interação entre o organismo e o ambiente, onde o indivíduo lança mão de um conjunto de estratégias destinadas a promover a adaptação frente às circunstâncias estressantes (BARBOSA; OLIVEIRA, 2008). Entre os diversos aspectos que influenciam as respostas às demandas e no enfrentamento das adversidades, encontra-se a percepçáo de contar com o apoio de pessoas significativas, o apoio social, como o apoio emocional ou prático dado pela família, por amigos e conhecidos, na forma de afeto, companhia, assistência e informação (FERREIRA, 2007).

Nesse sentido, estudos atuais buscam maior compreensão sobre os processos de adaptação e os fatores de proteção que estariam influenciando as diferentes respostas apresentadas às situaçóes estressoras pelos sujeitos e suas famílias.

Matsukura et al. (2007), considerando os efeitos protetores que $\mathrm{o}$ apoio social teria no enfrentamento das situaçóes estressoras, avaliaram a associação entre estresse e apoio social em mães de crianças com diferentes tipos de deficiência. O estudo envolveu uma amostra de 75 mães de crianças com idade entre 4 e 8 anos, com baixa renda e escolaridade, e os resultados indicaram elevada porcentagem de mães estressadas, que contam com menor número de pessoas suportivas quando comparadas às mães de crianças com desenvolvimento típico, além de indicarem uma associaçáo negativa entre o estresse $\mathrm{e}$ a satisfação com o apoio social. Os achados apoiam consideraçóes encontradas na literatura, de que máes ou cuidadores de familiares com comprometimentos crônicos de saúde possuem uma rede de apoio social menor do que as amostras de comparação e evidenciam o efeito protetivo do apoio social.

Revisão sistemática de literatura sobre o processo de adaptaçáo de pais de crianças com PC foi realizada por Rentinck et al. (2006) nas bases MEDLINE² e PsychINFO 3 , nas quais encontraram 22 estudos com divergências entre os desenhos de pesquisa, os instrumentos de coleta e as especificidades das populações investigadas. Contudo, foi possível extrair que os fatores relacionados às crianças (limitaçôes físicas, gravidade do comprometimento funcional, problemas de comportamento infantil), aos pais (saúde mental, recursos emocionais, circunstâncias pessoais, como desemprego, diferenças entre pais e mães, funcionamento e composição familiar) e ao contexto externo (apoio social, apoio formal dos serviços e de profissionais) estão envolvidos no processo de adaptação parental. Os autores concluem ainda que a adaptaçáo dos pais pode mudar em 
função da fase de desenvolvimento da criança e da vida da família ao longo do tempo.

Diante do exposto, o presente estudo objetiva descrever os níveis de estresse e o apoio social percebido de cuidadores de crianças com paralisia cerebral, assim como investigar relaçóes entre estresse e apoio social e variáveis relativas aos cuidadores, ao ambiente e às crianças, a saber: número de filhos, escolaridade do cuidador, renda familiar, temperamento e nível motor da criança.

Destaca-se como justificativa do estudo a tentativa de ampliar o conhecimento da área e agregar informaçóes sobre diferentes fatores na dimensão familiar, da criança e do ambiente que possam relacionar-se à presença de estresse e à forma como os cuidadores de crianças com deficiência percebem o apoio social recebido. Além disso, considera-se que os resultados advindos deste estudo possam facilitar e tornar mais eficaz o direcionamento de ações de saúde e educação, como a prestação de serviços por profissionais da área, com atuaçôes que considerem mais amplamente os contextos familiares em intervenções voltadas a essa população.

\section{Método}

\subsection{Participantes}

Participaram 50 crianças com paralisia cerebral na faixa etária de 3 a 7,5 (5,6 $\pm 1,51)$ anos de idade, os respectivos 50 cuidadores $^{4}$ principais das crianças com PC e 25 profissionais da reabilitação (Terapia Ocupacional e Fisioterapia) responsáveis pelo tratamento das crianças em seis instituiçôes de saúde de quatro cidades de médio porte do interior do estado de São Paulo.

A amostra participante foi intencional, sendo identificada com objetivo e por conveniência (PAGANO; GAUVREAU, 2004).

As crianças, de ambos os sexos, apresentavam diferentes tipos de PC, com predomínio de tetraparesia (66\%) e espasticidade (70\%). Em relação ao nível motor, as crianças estavam classificadas no nível I (24\%), II (12\%), III (6\%), IV (22\%) e V (36\%), de acordo com o Sistema de Classificação da Funçáo Motora Grossa para Paralisia Cerebral - GMFCS.

Acerca dos cuidadores, todos os entrevistados são do sexo feminino, em $94 \%$ dos casos é a mãe das crianças, nos outros $6 \%$ é a avó das crianças. A idade média foi de $34,3( \pm 8,8)$ anos, sendo que a maioria é casada (54\%), vive com companheiro (78\%), tem dois filhos (42\%), e declarou renda familiar de até dois salários mínimos (68\%); apenas $14 \%$ da amostra está no mercado de trabalho. Sobre a escolaridade, a maior parte (44\%) possui o ensino fundamental e $42 \%$ têm o ensino médio, sendo que as demais não são alfabetizadas (8\%) ou possuem ensino superior (6\%).

Quanto aos profissionais, a idade média foi de 35 $( \pm 8)$ anos, entre fisioterapeutas $(56 \%)$ e terapeutas ocupacionais (44\%), com vínculo empregatício médio de $7( \pm 7)$ anos nas instituições em que tratam das crianças. Ressalta-se que a participação dos profissionais da reabilitação no estudo possibilitou a aquisição de informações técnicas mais precisas relativas à manifestaçáo clínica da paralisia cerebral nas crianças (dado de caracterização da amostra) e do nível de função motora delas (variável da criança que foi investigada em sua relação com o estresse e apoio social dos cuidadores).

\subsection{Instrumentos}

\subsubsection{Ficha de identificação dos participantes}

Foi elaborada e utilizada uma ficha de identificação dos participantes para caracterização da amostra do estudo, contendo dados pessoais dos cuidadores, das crianças e dos profissionais, da manifestação clínica da paralisia cerebral, do atendimento da criança e do temperamento dela.

\subsubsection{Sistema de Classificação da Função Motora Grossa para Paralisia Cerebral (GMFCS)}

Para avaliar o nível motor das crianças foi utilizado o GMFCS de Palisano et al. (1997) em sua versão traduzida e adaptada para o português por Hiratuka, Matsukura e Pfeifer (2010). Esse instrumento fundamenta-se no critério de que distinçôes na função motora entre os níveis propostos são significativas clinicamente e baseadas nas limitaçóes funcionais e na necessidade de tecnologia assistiva, com ênfase para o movimento iniciado de forma voluntária, particularmente o sentar e o andar. A avaliação é feita através de observação das habilidades motoras grossas da criança, classificando-a em um dos cinco níveis da escala ordinal (I, II, III, IV ou V), que são dependentes da idade (antes dos 2 anos, entre 2 e 4 anos, entre 4 e 6 anos e entre 6 e 12 anos) (PALISANO et al., 1997). 


\subsubsection{Inventário de Sintomas de Stress para Adultos de Lipp (ISSL)}

Com o intuito de identificar se os cuidadores possuem sintomas de estresse foi empregado o ISSL em sua versão mais recente, após autorização da autora (LIPP, 2000). O ISSL é útil na identificação de quadros característicos do estresse, sendo possível apontar a fase do estresse em que a pessoa se encontra (alerta, resistência, quase exaustão ou exaustão) e a área de maior vulnerabilidade, ou seja, o tipo de sintoma que se manifesta na pessoa (físico ou psicológico) (LIPP, 2000).

\subsubsection{Questionário de Suporte Social (SSQ)}

Para avaliar o apoio social dos cuidadores foi utilizado o SSQ de Saranson et al. (1983) traduzido e validado no Brasil por Matsukura, Marturano e Oishi (2002). O SSQ é formado por questôes relativas aos apoios emocional e instrumental, permitindo identificar o número de pessoas que o respondente percebe como fonte de apoio e a satisfação que tem com o apoio percebido (SARANSON et al., 1983).

\subsection{Procedimentos de coleta}

Por tratar-se de um estudo que envolve seres humanos, todos os procedimentos éticos vigentes foram cumpridos. O projeto de pesquisa foi submetido e aprovado pelo Comitê de Ética em Pesquisa em Seres Humanos da Universidade Federal de São Carlos, sob protocolo de número 149/2010, segundo os termos da portaria 196/1996, sendo que todos os cuidadores e profissionais foram informados sobre os objetivos do estudo e, após confirmação de seu interesse em participar, todos assinaram o termo de consentimento livre e esclarecido.

Em um local disponibilizado pelos serviços, durante o horário de atendimento das crianças, aconteceu a coleta individual com os cuidadores, aos quais foram aplicados os instrumentos ${ }^{5}$ (ficha de identificação do cuidador/criança, o ISSL e o SSQ), através de entrevista estruturada.

O profissional da reabilitação que atendia a criança no serviço foi contatado em seguida para responder a ficha de identificaçáo do profissional/criança e o GMFCS, através de autoaplicação. Os dados da ficha de identificação do profissional/criança e do GMFCS respondidos pelos profissionais serviram para caracterizaçáo da amostra de crianças do presente estudo e para a obtenção do nível de funçâo motora de cada criança. $\mathrm{Na}$ ocasião da coleta, instruçóes prévias acerca do preenchimento dos instrumentos foram fornecidas.

\subsection{Análise dos dados}

Para análise dos dados foram realizados: categorização e quantificação das informaçóes obtidas por meio da ficha de identificação dos participantes; resumo descritivo das características da amostra e dos dados obtidos por meio de GMFCS, ISSL e SSQ; relaçôes entre as variáveis investigadas por meio dos testes Qui-quadrado, Exato de Fisher, Mann-Whitney, Kruskal-Wallis e Odds-Ratio.

Os resultados dos testes utilizados nas análises foram considerados com significância quando o valor associado à estatística que o descreve (p-valor) foi inferior a 0,05 .

Consideram-se as variáveis investigadas no estudo como presentes em diferentes dimensóes: variáveis do cuidador (apoio social, estresse e escolaridade), da criança (nível de função motora e temperamento) e do ambiente (número de filhos e renda familiar).

\section{Resultados}

A partir da aplicação do ISSL foi inicialmente identificada a presença ou ausência de estresse nos cuidadores participantes. Em seguida, no caso da presença de estresse, identificou-se a fase na qual se encontrava (alerta, resistência, quase exaustão ou exaustão) e, finalmente, o tipo de sintoma de estresse presente (físico ou psicológico).

Na Tabela 1 apresenta-se o número de cuidadores em que foram encontrados sintomas de estresse e como ele manifestava-se.

Revelou-se, assim, que entre o total de cuidadores com estresse $(n=33)$, nenhum se encontra na fase

Tabela 1. Manifestação do estresse entre os cuidadores.

n $\%$

\begin{tabular}{lrr}
\hline Apresentou sintomas de estresse & & \\
Sim & 33 & 66,0 \\
$\quad$ Não & 17 & 34,0 \\
Fase do estresse & & \\
$\quad$ Resistência & 31 & 93,9 \\
$\quad$ Quase exaustão & 2 & 6,1 \\
Sintoma predominante & & \\
$\quad$ Físicos & 8 & 24,2 \\
$\quad$ Psicológicos & 23 & 69,7 \\
Físicos e psicológicos & 2 & 6,1 \\
\hline
\end{tabular}


de alerta (I) ou na fase de exaustáo (IV), mas que estão concentrados nas fases intermediárias, de resistência (II) e de quase exaustão (IV), estando a maioria na fase II do estresse (93,9\%), apresentando estresse de tipo psicológico (69,7\%).

Através do SSQ, acerca do número de pessoas que os cuidadores consideram como fonte de apoio social (SSQ-N), foram encontrados resultados que revelaram a média de 2,07 e, para a satisfação dos cuidadores com o apoio percebido (SSQ-S), a média de 5,45, conforme apresentado na Tabela 2 .

A partir da aplicação do SSQ, além dos índices $\mathrm{N}$ e $\mathrm{S}$, foi possível identificar as fontes de apoio social dos cuidadores e a frequência com que são citadas ao longo do instrumento, como pode ser visto na Tabela 3.

Nota-se que os cuidadores participantes reconhecem pais e irmáos $(38,2 \%)$ como maiores fontes de apoio, em seguida apontam os filhos (20,2\%) e, em terceiro lugar, o cônjuge (15,2\%). Os resultados também indicam que $85,91 \%$ do total de pessoas citadas como fonte de apoio eram familiares.

Tabela 2. Distribuição dos cuidadores segundo o apoio social.

\begin{tabular}{cc}
\hline SSQ & Resumo descritivo $^{(1)}$ \\
\hline SSQ-N & $2,07 \pm 1,16 ; 1,85 ; 0,74 / 6,30$ \\
SSQ-S & $5,45 \pm 0,83 ; 5,75 ; 2,11 / 6,00$ \\
\hline
\end{tabular}

${ }^{(1)}$ Resumo descritivo em média, desvio padrão, mediana, mínimo e máximo.

Tabela 3. Fontes de apoio social dos cuidadores.

\begin{tabular}{lcc}
\hline \multicolumn{1}{c}{ Fonte de apoio social } & $\mathbf{n}$ & $\mathbf{0}$ \\
\hline Pais e irmãos & 1.039 & 38,2 \\
Filhos & 548 & 20,2 \\
Marido & 412 & 15,2 \\
Família extensa $^{(1)}$ & 311 & 11,4 \\
Amigos & 309 & 11,4 \\
Vizinhos/namorados/compadres & 85 & 3,1 \\
Profissionais de saúde/pastor/chefe & 15 & 0,6 \\
\hline Total & $\mathbf{2 . 7 1 9}$ & $\mathbf{1 0 0}$ \\
\hline
\end{tabular}

${ }^{(1)}$ Avós, tios, sobrinhos, primos, netos, sogros e cunhados.
A seguir apresentam-se as análises que buscaram verificar relaçóes entre variáveis investigadas do cuidador, da criança e do ambiente.

Através dos testes Qui-quadrado e Exato de Fisher constatou-se não existir associação significativa entre níveis de função motora da criança (leve e grave) ${ }^{6} \mathrm{e}$ características do estresse dos cuidadores (GMFCS e sintoma de estresse, $p=0,869$; GMFCS e fase de quase exaustão, $\mathrm{p}=1,000$; GMFCS e sintoma físico, $\mathrm{p}=0,248$; GMFCS e sintoma psicológico, $\mathrm{p}=0,676$ ), assim como, através do teste de Mann-Whitney, não houve associação significativa entre níveis de funçáo motora da criança e características do apoio social dos cuidadores (SSQ-S e GMFCS, $\mathrm{p}=0,168$; SSQ-Family e GMFCS, $\mathrm{p}=0,737$ ).

Ao investigar as características do estresse (presença ou não de estresse, fase do estresse e predominância do tipo de sintoma) com algumas variáveis do cuidador, do ambiente e da criança, foi possível identificar, através da análise de regressão logística (teste Odds-Ratio), que náo houve associação significativa do estresse com o temperamento (agradável, difícil ou instável) da criança ( $\mathrm{p}=0,503)$, com a escolaridade $(\mathrm{p}=0,540)$, com o número de filhos ( $\mathrm{p}=0,152)$ do cuidador e com a renda familiar $(\mathrm{p}=0,155)$.

Quando investigada associação entre as características do apoio social (número de pessoas suportivas, satisfação com o apoio percebido e percentual de familiares suportivos) e as variáveis do cuidador, ambiente e criança, foi encontrada associação significativa apenas da escolaridade do cuidador com o número de pessoas suportivas, através do teste de Mann-Whitney, como apresentado na Tabela 4.

Observa-se que a escolaridade relacionou-se com o número de pessoas suportivas, sendo que $50 \%$ dos cuidadores com o ensino fundamental possuem mais de 2,16 pessoas suportivas, enquanto que cerca de $50 \%$ dos cuidadores com ensino médio têm até 1,44 pessoas suportivas.

De acordo com o teste de Kruskal-Wallis, náo houve associaçóes entre as características do apoio social com as demais variáveis investigadas do

Tabela 4. Comparação entre escolaridades de acordo com o número de pessoas suportivas.

\begin{tabular}{ccccc}
\hline & & $\begin{array}{c}\text { Ensino fundamental } \\
(\mathbf{n}=\mathbf{2 2})\end{array}$ & $\begin{array}{c}\text { Ensino médio } \\
(\mathbf{n}=\mathbf{2 1})\end{array}$ & $\mathbf{p}^{(1)}$ \\
\hline & Média \pm desvio padrão & $2,35 \pm 1,23$ & $1,71 \pm 0,90$ & \\
SSQ-N & Mediana & 2,16 & 1,44 & $0,037^{*}$ \\
& $\left(1^{\circ}\right.$ quartil; $3^{\circ}$ quartil $)$ & $(1,58 ; 2,82)$ & $(0,96 ; 2,19)$ & \\
\hline & Mínimo/máximo & $0,90 / 6,30$ & $0,74 / 3,67$ & \\
\hline
\end{tabular}

${ }^{*} \mathrm{p}<0,05$ (1) $^{(1)}$ este de Mann-Whitney. 
Tabela 5. Associação entre apoio social e estresse dos cuidadores.

\begin{tabular}{cccccc}
\hline Apoio social & Efeito & Erro padrão & OR $^{(1)}$ & p & $\begin{array}{c}\text { IC } \\
(\text { OR; } 95 \%)^{(2)}\end{array}$ \\
\hline SSQ-N & $-0,81$ & 0,34 & 0,44 & $0,017^{*}$ & $(0,23-0,86)$ \\
SSQ-S & $-0,93$ & 0,77 & 0,82 & 0,619 & $(0,38-1,77)$ \\
SSQ-Family & $-0,09$ & 0,05 & 0,98 & 0,171 & $(0,94-1,01)$ \\
\hline
\end{tabular}

${ }^{*} \mathrm{p}<0,05 ;{ }^{(1)}$ teste Odds-Ratio - OR = 1 significa que não há relação entre X e Y, OR > 1, a chance de Y acontecer aumenta com o aumento de $\mathrm{X}, \mathrm{OR}<1$, a chance de $\mathrm{Y}$ acontecer diminui com o aumento de $\mathrm{X}$; ${ }^{(2)}$ intervalo do Odds-Ratio com nível de confiança de $95 \%$.

cuidador, ambiente e criança: temperamento da criança e número de pessoas suportivas ( $\mathrm{p}=0,487)$; temperamento da criança e satisfação com o apoio percebido $(\mathrm{p}=0,347)$; temperamento da criança e percentual de familiares suportivos $(\mathrm{p}=0,148)$. Através do teste de Kruskal-Wallis também não houve associação significativa entre o número de filhos e os escores do apoio recebido, entre o número de filhos e os escores da satisfação e entre o número de filhos e o percentual de familiares suportivos ( $\mathrm{p}=0,997, \mathrm{p}=0,938$ e $\mathrm{p}=0,652$, respectivamente).

Da mesma forma, através do teste Mann-Whitney não se verificou associação significativa entre renda familiar e categorias do apoio social (SSQ-N, $\mathrm{p}=0,980 ;$ SSQ-S, $\mathrm{p}=0,321$; SSQ-Family, $\mathrm{p}=0,104)$.

Quanto à associação entre apoio social e estresse dos cuidadores, os resultados são apresentados na Tabela 5, de acordo com o modelo de regressáo logística ajustado para explicar o estresse em função de características do apoio social.

A partir das estimativas pontuais e intervalares do teste Odds-Ratio, os achados evidenciam uma redução da chance do aparecimento de sintomas de estresse na medida em que aumenta o número de pessoas suportivas.

\section{Discussão}

No presente estudo procurou-se descrever o estresse e o apoio social percebido de cuidadores de crianças com PC, além de investigar relaçóes entre variáveis dos cuidadores, das crianças e do ambiente na tentativa de buscar dados sobre fatores que possam relacionar-se ao estresse e ao apoio social percebido por esses cuidadores.

Em relação à condição de estresse nos cuidadores participantes do estudo, foi encontrado que a maioria (66\%) apresenta sintomas de estresse, estando a maior parte deles na fase de resistência e com predominância de sintomas psicológicos. Apesar de a porcentagem de cuidadores com estresse não ter sido tão elevada, quando comparada, por exemplo, aos estudos de
Matsukura (2001), que encontrou estresse em $88 \%$ da amostra de máes de crianças com deficiência, aos de Nunes (2010), em que a ocorrência de estresse foi observada em $76 \%$ da amostra de mães de crianças com deficiência intelectual, e aos de Souza (2010), com incidência de estresse em $91 \%$ da amostra de mães de crianças com cardiopatia congênita, o achado reafirma a consideração de que mães de crianças com algum comprometimento no desenvolvimento comumente estão estressadas (GLENN et al., 2008; MANUEL et al., 2003). Tal achado é explicado, possivelmente, pela sobrecarga de cuidados com a criança, pela forma com que lidam e se adaptam ao processo de ter na família uma criança com alteraçóes no desenvolvimento, assim como pela limitação nos papéis ocupacionais que desempenham, o que reforça a importância de os profissionais e programas de saúde/educação voltados a esses cuidadores darem maior atenção a eles.

Os achados relativos à fase e ao tipo de sintoma do estresse predominante foram semelhantes aos das pesquisas de Souza (2010), Nunes (2010) e Matsukura et al. (2007), lembrando que na fase de resistência o organismo entra em ação para impedir o desgaste total de energia, resistindo aos agentes estressores na tentativa inconsciente de restabelecer o equilíbrio interior, com prevalência da sensação de desgaste generalizado (SOUZA, 2010). Esse resultado sugere, segundo discutem Matsukura et al. (2007), um estilo de vida potencialmente estressante dessas mulheres, o que é bastante preocupante, na medida em que se nada for feito para remediar esse processo e se não conseguirem enfrentar a situação estressora, ou se a mesma náo for retirada, essas cuidadoras somente conseguirão sair da fase de resistência para outra mais grave. Tais consideraçóes reforçam a importância de ações que busquem auxiliar as mães a enfrentarem com sucesso o estresse do cotidiano.

No que se refere ao apoio social, o índice SSQ-N foi encontrado como relacionado à variável escolaridade do cuidador, indicando que os cuidadores com maior nível escolar (ensino médio) contam com um menor 
número de pessoas suportivas que os de menor nível de escolaridade (ensino fundamental).

Também se verificou o baixo número de pessoas percebidas pelos cuidadores como fontes de apoio e alta satisfação (quando comparadas as média do SSQ-N e do SSQ-S com os escores máximos do instrumento, 9 e 6 , respectivamente). Tais achados apresentam-se convergentes aos de outros estudos que utilizaram o SSQ para investigação do apoio social das mães (CID, 2008; MATSUKURA et al., 2007; MATSUKURA, 2001). Possivelmente, esses dados podem estar relacionados à hipótese de que cuidadores de crianças com deficiência, por dedicarem a maior parte do seu tempo e atividades diárias aos filhos, com restriçôes em seus papéis ocupacionais e contatos sociais, não têm condiçóes de vivenciar uma rede de apoio ampliada. Em relação à satisfação com o apoio que recebem, mesmo que essa rede seja composta por um pequeno número de pessoas, observa-se que a satisfação indicada é bastante alta.

Com relação às fontes de apoio social, observou-se que os cuidadores consideram como principais fontes pais e irmãos, seguidos dos filhos e, depois, dos cônjuges. Esse achado distancia-se de outros estudos brasileiros que utilizaram o instrumento SSQ e indicaram como principal fonte de apoio o cônjuge (FERREIRA, 2007; MATSUKURA; MARTURANO; OISHI, 2002).

Ferreira (2007), ao estudar aspectos familiares envolvidos no desenvolvimento de crianças com PC, obteve que o marido representou $21 \%$ das 1.046 fontes citadas pelas máes, enquanto no estudo de adaptação transcultural do SSQ de Matsukura, Marturano e Oishi (2002), para mães de crianças típicas, os maridos representaram 22\% das 6.180 citaçôes. Sendo alta a incidência das cuidadoras que vivem com companheiro (78\%) e são casadas (54\%) na amostra do presente estudo, esse achado chama a atenção.

Observa-se que o período de tempo que separa tais estudos e a velocidade nas modificaçôes e arranjos no cotidiano familiar podem ser apontados como possíveis fatores que auxiliam na compreensão desses resultados. A princípio, hipotetiza-se que as transformaçôes sociais ligadas ao gênero, no que diz respeito ao ingresso da mulher no mercado de trabalho, sua estabilização e independência financeira/afetiva possam afetar a rede de apoio das cuidadoras e/ou sua percepçáo quanto ao apoio que lhes é ofertado pelo companheiro.

Não obstante, a família como um todo, seja ela próxima ou extensa, foi apontada como a principal fonte de apoio social, o que reafirma a importância do papel dos familiares como parte de uma unidade social a ser provida e como auxílio para manter a saúde de mães e dos demais membros do grupo familiar (FERREIRA, 2007).

Ainda no que se refere às fontes de apoio social, merece atenção a presença dos profissionais da saúde nas citaçôes dos cuidadores, mesmo que de forma discreta. De maneira semelhante, Matsukura et al. (2007), ao observarem que profissionais foram igualmente incluídos como fonte de apoio em ambos os grupos investigados (mães de crianças com deficiência e mães de crianças com desenvolvimento típico), sugerem que o resultado encontrado pode indicar que esses profissionais são fonte de apoio emocional para essas mães, na medida em que o SSQ é um instrumento que avalia mais claramente o apoio social do tipo emocional e instrumental, não sendo sensível para a avaliação do apoio social do tipo informacional, no qual provedores de serviços, como os de saúde e educação, geralmente são relacionados.

Considera-se que investigações mais específicas, que focalizem os tipos de apoio social fornecidos pelos profissionais da saúde aos familiares e tipos de apoio esperados pelos familiares e vindos desses mesmos profissionais poderiam revelar aspectos importantes desse processo e muito contribuiriam para as práticas em saúde e estudos na área.

Destaca-se que na análise de associaçóes entre as variáveis investigadas não foi encontrada nenhuma relação entre características sociodemográficas, de saúde e comportamento da criança e o estresse do cuidador. Para melhor compreender esse resultado, estudos futuros envolvendo amostras maiores poderiam fornecer mais elementos para verificar essas possíveis relaçóes na realidade que se pretende alcançar. Os resultados encontrados não são apoiados pelo estudo de Raina et al. (2005), pesquisa de referência na área e que objetivou identificar determinantes de saúde física/psicológica de cuidadores de crianças com PC, sendo essa fortemente influenciada pelo comportamento das crianças e pela demanda de cuidados. Além disso, a renda das famílias, quando associada a demais aspectos analisados, teve efeitos indiretos sobre a saúde psicológica e física dos cuidadores.

Nessa direção, Sipal et al. (2010) também localizaram associação positiva do estresse e do apoio social dos cuidadores com problemas comportamentais das crianças com PC, indicando que o contexto familiar desempenha um papel importante no comportamento infantil e que esse último também é importante para o bem-estar psicológico/emocional dos cuidadores, o que, na 
busca de dados úteis às estratégias de prevenção, vigilância e assistência aos cuidadores de crianças com deficiência, reforça a necessidade de continuar a investigar fatores que influenciam a saúde de familiares.

Os resultados da associaçáo entre o apoio social e o estresse encontrados no presente estudo evidenciam a relação entre esses fatores, na medida em que o aumento do número de pessoas como fonte de apoio (SSQ-N) está relacionado com a redução nas chances de aparecimento do estresse, o que corrobora a literatura e sustenta o conceito do apoio social como um dos mais importantes recursos de proteção contra o estresse (NUNES, C. C., 2010; MATSUKURA et al., 2007; SKOK; HARVEY; REDDIHOUGH, 2006). Não obstante, no presente estudo não foi observada relação entre a satisfação com o apoio social e o estresse, como na pesquisa de Matsukura et al. (2007), em que foi observada associação negativa entre o estresse e a satisfação com o apoio social (índice SSQ-S).

Considerando-se a complementaridade desses achados acerca das relaçóes dos índices de apoio social e estresse, sugere-se que as intervençóes, quando direcionadas às famílias, devam investir na quantidade e na qualidade do apoio social, ou seja, no aumento da rede de apoio e na compreensão e satisfação advindas desses relacionamentos.

\section{Considerações finais}

Os resultados apresentados acrescentam ao conhecimento da área ao evidenciarem como tendência, na amostra investigada, a presença de estresse entre cuidadores de crianças com paralisia cerebral, mesmo que em diferentes níveis, assim como baixo apoio social percebido por essa população alvo em termos de número de pessoas suportivas, simultaneamente a uma adequada satisfação acerca do apoio recebido.

Considera-se a relevância da investigação ao reforçar a relação entre as variáveis estresse e apoio social dos cuidadores, o que sustenta o conceito do apoio social como importante fator de proteçáo contra os sintomas de estresse. Por outro lado, entre as demais variáveis estudadas não foi possível identificar relaçôes (exceto escolaridade versus apoio social), o que distancia este de alguns outros estudos que as sugerem. Assim, entende-se como relevante a continuidade das investigaçóes com cuidadores de crianças com PC, na direção de ampliar o conhecimento acerca da influência da condição da criança com comprometimento e do contexto ambiental na saúde da família.

Através do desenho metodológico traçado, com todas as nuanças e cuidados possíveis de adotarem-se, buscou-se uma representação provisória da realidade que se desejava apreender, baseando-se na interlocução dos resultados encontrados com outros conhecimentos. Assim, os achados do presente estudo são sinalizadores importantes da realidade, que podem ser aprofundados e/ou confirmados em pesquisas posteriores. Todavia, aponta-se que os dados obtidos devem ser compreendidos diante do limite apresentado nesta investigação, que se refere ao tamanho amostral.

Por fim, considera-se que o estudo oferece subsídios para o desenvolvimento de novas investigaçóes e para a reflexão e o planejamento de açóes em diferentes níveis, direcionadas às crianças com deficiência e respectivas famílias. Também permite pensar estratégias de atenção para além da criança com deficiência, direcionando o olhar para os envolvidos no cuidar dessas crianças, seus cuidadores/familiares, que são participantes fundamentais no processo de desenvolvimento infantil e que também necessitam de cuidados, atenção e de se sentirem bem - o que muitas vezes não é permitido pelo caminho que percorrem junto ao filho com deficiência.

\section{Agradecimentos}

As autoras agradecem aos cuidadores, crianças, profissionais e instituições pela participação no estudo, ao apoio da Coordenação de Aperfeiçoamento de Pessoal de Nível Superior (CAPES) e ao consultor estatístico Hélio Rubens pela contribuição na análise dos dados.

\section{Referências}

ARAGĀO, E. I. S. et al. Suporte social e estresse: uma revisão da literatura. Psicologia em Foco, Aracaju, v. 2, n. 1, p. 79-90, 2009.

BARBOSA, A. J. G.; OLIVEIRA, L. D. Estresse e enfrentamento em pais de pessoas com necessidades especiais. Psicologia em Pesquisa, Juiz de Fora, v. 2, n. 2, p. 36-50, 2008.

BYRNE, M. B. et al. Health status of caregivers of children with cerebral palsy. Child: Care, Health and Development, Oxford, v. 36, n. 5, p. 696-702, 2010. PMid:20074250. http://dx.doi.org/10.1111/j.1365-2214.2009.01047.x

CALDERON, R.; GREENBERG, M. T. Stress and coping in hearing mothers of children with hearing loss: factors affecting mother and child adjustment. American Annals of the Deaf, Washington, v. 144, n. 1, p. 7-18, 1999. http://dx.doi.org/10.1353/aad.2012.0153 
CAMARGOS, A. C. R. et al. Avaliação da sobrecarga do cuidador de crianças com paralisia cerebral através da escala Burden interview. Revista Brasileira de Saúde Materno Infantil, Recife, v. 9, n. 1, p. 31-37, 2009. http:// dx.doi.org/10.1590/S1519-38292009000100004

CANNING, R. D.; HARRIS, E. S.; KELLEHER, K. J. Factors predicting distress among caregivers to children with chronic medical conditions. Journal of Pediatrics Psychology, Atlanta, v. 21, n. 5, p. 735-749, 1996. http:// dx.doi.org/10.1093/jpepsy/21.5.735

CID, M. F. B. Fatores de risco e proteção: saúde mental de mães e filhos, suporte social e estilo parental. 2008. $182 \mathrm{f}$. Dissertação (Mestrado em Educação Especial)Universidade Federal de São Carlos, São Carlos, 2008. CROWE, T. K.; VANLEIT, B.; BERGHMANS, K. K. Mothers' perceptions of child care assistance: the impact of a child's disability. American Journal of Occupational Therapy, Bethesda, v. 54, n. 1, p. 52-58, 2000. http:// dx.doi.org/10.5014/ajot.54.1.52

DANTAS, M. S. A. et al. Impacto do diagnóstico de paralisia cerebral para a família. Revista Texto e Contexto Enfermagem, Santa Catarina, v.19, n. 2, p. 229-237, 2010. http://dx.doi.org/10.1590/S0104-07072010000200003

DAVIS, E. et al. The Impact of caring for a child with cerebral palsy: quality of life for mothers and fathers. Child: Care, Health and Development, Oxford, v. 36, n. 1, p. 63-73, 2009. PMid:19702639. http://dx.doi. org/10.1111/j.1365-2214.2009.00989.x

FERRARI, J. P.; MORETE, M. C. Reações dos pais diante do diagnóstico de paralisia cerebral em crianças com até 4 anos. Cadernos de Pós-Graduação em Distúrbios do Desenvolvimento, São Paulo, v. 4, n. 1, p. 25-34, 2004.

FERREIRA, H. B. G. Aspectos familiares envolvidos no desenvolvimento de crianças com paralisia cerebral. 2007. 110 f. Dissertação (Mestrado em Saúde da Comunidade)-Universidade de São Paulo, Ribeirão Preto, 2007.

GLENN, S. et al. Maternal parenting stress and its correlates in families with a young child with cerebral palsy. Child: Care, Health and Development, Oxford, v. 35, n. 7, p. 71-78, 2008.

HEIMAN, T. Parents of children with disabilities: resilience, coping and future expectations. Journal of Developmental and Physical Disabilities, Heidelberg, v. 14, n. 2, p. 159-171, 2002. http://dx.doi. org/10.1023/A:1015219514621

HIRATUKA, E. Demandas de mães de crianças com paralisia cerebral em diferentes fases do desenvolvimento infantil. 2009. 298 f. Dissertação (Mestrado em Educação Especial)-Universidade Federal de São Carlos, São Carlos, 2009.

HIRATUKA, E.; MATSUKURA, T. S.; PFEIFER, L. I. Adaptação transcultural para o Brasil do sistema de classificação da função motora grossa (GMFCS). Revista Brasileira de Fisioterapia, São Carlos, v. 14, n. 6, p. 537-544, 2010. http://dx.doi.org/10.1590/ S1413-35552010000600013
KOKKONEN, J. et al. Social outcome of handicapped children as adults. Developmental Medicine and Child Neurology, Oxford, v. 33, p. 1095-1100, 2001. http:// dx.doi.org/10.1111/j.1469-8749.1991.tb14832.x

LIN, J.; ZEBRACK, B. Caring for family members with chronic physical illness: a critical review of caregiver literature. Health and Quality Life Outcomes, London, v. 2, p. 1-9, 2004. PMid:14713317 PMCid:PMC333432. http://dx.doi.org/10.1186/1477-7525-2-1

LIPP, M. E. N. O Modelo quadrifásico do stress. In: LIPP, M. E. N. Mecanismos neuropsicofisiológicos do stress: teoria e aplicaçóes clínicas. São Paulo: Casa do Psicólogo, 2003. p. 17-22.

LIPP, M. E. N. Manual do inventário de sintomas de stress para adultos de Lipp (ISSL). São Paulo: Casa do Psicólogo, 2000.

MANCINI M. C. et al. Gravidade da paralisia cerebral e desempenho funcional. Revista Brasileira de Fisioterapia, São Carlos, v. 8, n. 3, p. 253-260, 2004.

MANUEL, J. et al. Stress and adaptation in mothers of children with cerebral palsy. Journal of Pediatrics Psychology, Atlanta, v. 28, n. 3, p. 197-201, 2003. http:// dx.doi.org/10.1093/jpepsy/jsg007

MATSUKURA, T. S. Mães de crianças com necessidades especiais: stress e percepção de suporte social. 2001. 170 f. Tese (Doutorado em Saúde Mental)-Universidade de São Paulo, Ribeirão Preto, 2001.

MATSUKURA, T. S. et al. Estresse e suporte social em mães de crianças com necessidades especiais. Revista Brasileira de Educação Especial, São Carlos, v. 13, n. 3, p. 415-428, 2007. http://dx.doi.org/10.1590/ S1413-65382007000300008

MATSUKURA, T. S.; MARTURANO, E. M.; OISHI, J. O Questionário de suporte social (SSQ): estudos da adaptação para o português. Revista Latino-Americana de Enfermagem, Ribeirão Preto, v. 10, n. 5, p. 675-681, 2002. http://dx.doi.org/10.1590/S0104-11692002000500008

MATSUKURA, T. S.; SIME, M. M. Demandas e expectativas de famílias de crianças com necessidades especiais: de situaçóes do cotidiano aos técnicos envolvidos no tratamento. Temas sobre Desenvolvimento, São Paulo, v. 16, n. 94, p. 214-221, 2008.

NUNES, C. C. Famílias de crianças em idade escolar com deficiência intelectual, dificuldades de aprendizagem ou desenvolvimento típico: comportamento, estresse materno, apoio social e percepção de impacto familiar. 2010. $106 \mathrm{f}$. Tese (Doutorado em Psicologia)-Universidade de São Paulo, Ribeirão Preto, 2010.

NUNES, A. M. S. O Perfil do cuidador da criança portadora de paralisia cerebral. Revista Meio Ambiente e Saúde, Manhuaçu, v. 2, n. 1, p. 1-21, 2007.

PAGANO, M.; GAUVREAU, K. Princípios de bioestatística. São Paulo: Cengage Learning, 2004.

PALISANO, R. et al. Developmental and reliability of a system to classify gross motor function in children with cerebral palsy. Developmental Medicine and Child Neurology, Oxford, v. 39, n. 4, p. 214-223, 1997. http:// dx.doi.org/10.1111/j.1469-8749.1997.tb07414.x 
RAINA, P. et al. The health and well-being of caregivers of children with cerebral palsy. Pediatrics, Ilinois, v. 115, n. 6, p. 626-636, 2005.

RENTINCK, I. C. M. et al. Parents of children with cerebral palsy: a review of factors related to the process of adaptation. Child: Care, Health and Development, Oxford, v. 33, n. 2, p. 161-169, 2006. PMid:17291320. http://dx.doi.org/10.1111/j.1365-2214.2006.00643.x

ROSEMBAUM, P. et al. A report: the definition and classification of cerebral palsy abril 2006. Developmental Medicine and Child Neurology, Oxford, v. 49, n. 109, p. 8-14, 2007.

SARASON, I. G. et al. Assessing social support: the social support questionnaire. Journal of Personality and Social Psychology, Washington, n. 44, p. 127-139, 1983. http://dx.doi.org/ 10.1037/0022-3514.44.1.127

SIPAL, R. F. et al. Course of behavior problems of children with cerebral palsy: the role of parental stress and support. Child: Care, Health and Development, Oxford, v. 36, n. 1, p. 74-84, 2010. PMid:19702640. http://dx.doi.org/10.1111/j.1365-2214.2009.01004.x
SKOK, A.; HARVEY, D.; REDDIHOUGH, D. Perceived stress, perceived social support, and wellbeing among mothers of school-aged children with cerebral palsy. Journal of Intellectual and Developmental Disability, London, v. 31, n. 1, p. 53-57, 2006. PMid:16766323. http://dx.doi.org/10.1080/13668250600561929

SOUZA, D. S. B. Avaliação de estresse e enfrentamento das mães de crianças com cardiopatias congênitas. 2010. $152 \mathrm{f}$. Dissertação (Mestrado em Bases Gerais da Cirurgia)Universidade Estadual Paulista Júlio de Mesquita Filho, Botucatu, 2010.

STENER-VICTORIN, E. et al. Comparison of impact on mood, health, and daily living experiences of primary caregivers of walking and non-walking children with cerebral palsy and provided community services support. European Journal of Paediatric Neurology, Leuven, v. 14, n. 3, p. 239-246, 2010.

VIEIRA, N. G. B. et al. O Cotidiano de mães com crianças portadoras de paralisia cerebral. Revista Brasileira de Promoção da Saúde, Fortaleza, v. 21, n. 1, p. 55-60, 2008. http://dx.doi.org/10.5020/18061230.2008.p55

\section{Contribuição dos Autores}

As autoras foram responsáveis pela elaboração do texto de forma conjunta. Alyne Kalyane Câmara de Oliveira trabalhou na concepção e redação do artigo. Thelma Simôes Matsukura atuou na revisão do texto e orientou a primeira autora no desenvolvimento da pesquisa.

\section{Notas}

${ }^{1}$ Este trabalho é parte da dissertação de mestrado intitulada "Repertório funcional de crianças com paralisia cerebral: a perspectiva de cuidadores e profissionais”, apresentada ao Programa de Pós-Graduação em Terapia Ocupacional da UFSCar.

${ }^{2}$ Medical Literature Analysis and Retrieval System Online é a base on-line de dados bibliográficos da Biblioteca Nacional de Medicina dos Estados Unidos.

${ }^{3}$ Banco de dados on-line dedicado à literatura das Ciências do Comportamento e Saúde Mental.

${ }^{4}$ Este termo, no estudo, refere-se à pessoa que apresenta maior proximidade com a criança, permanece mais tempo com ela e realiza a maior parte dos cuidados.

${ }^{5}$ Outros instrumentos foram aplicados na investigação que deu origem ao presente artigo, envolvendo outros objetivos além dos que este estudo focaliza.

${ }^{6}$ Nas análises que envolviam a função motora das crianças foi utilizada a categorização leve para níveis I e II do GMFCS e grave para níveis IV e V. 